\title{
Calcium homeostasis in women with non-metastatic breast cancer with osteoporosis after a single dose of denosumab: a pilot study
}

\author{
Konstantinos Toulis, ${ }^{1,2}$ Paschalia Iliadou, ${ }^{1}$ Stylianos Mandanas, ${ }^{1}$ Polyzo Kazila, ${ }^{3}$ \\ Efterpi Margaritidou, ${ }^{1}$ Konstantinos Georgopoulos, ${ }^{1}$ Kalliopi Pazaitou-Panayiotou ${ }^{1}$
}

\begin{abstract}
${ }^{1}$ Department of Endocrinology, Theagenio Cancer Hospital, 2 Department of Endocrinology, 424 General Military Hospital, ${ }^{3}$ Department of Clinical Chemistry, Theagenio Cancer Hospital; Thessaloniki, Greece
\end{abstract}

Dear Sir,

Along with achieving a reduction in the incidence of osteoporotic fractures, denosumab (a human monoclonal antibody against the receptor activator of nuclear factor kappa-B ligand (RANKL) causes conformational changes in the calcium-sensing receptor (CaSR), leading to parathyroid hormone exocytosis. In breast cancer cells, malignant transformation may be coupled with a change of CaSR functionality. ${ }^{1}$ It is still unknown whether this change is tissue-specific or a generalized one. To this end, we investigated the homeostatic response of calcium in women with nonmetastatic breast cancer following a single injection of denosumab.

Forty-six $(\mathrm{n}=46)$ female osteoporotic patients, eligible for denosumab, were prospectively enrolled

Key words: Breast cancer, Denosumab, Osteoporosis

Address for correspondence:

Dr. Kalliopi Pazaitou-Panayiotou MD, PhD, Department of Endocrinology, Theagenio Cancer Hospital, 2 Al. Simeonidi Str., 54007 Thessaloniki, Greece;

Tel.: +30 2310898379,2310898176 , Fax: +30 2310898809 ,

E-mail: kpazaitoupanayiotou@gmail.com

Received: 07-06-2016, Accepted: 09-06-2016 from the outpatient clinics during 2014. Patients were divided into two groups. Group A included postmenopausal patients with a concurrent diagnosis of non-metastatic breast cancer $(n=21)$. Group B included 25 postmenopausal women without a history of malignant disease (controls). Specific exclusion criteria were applied to ensure that no medications or diseases affecting bone metabolism were present. After baseline assessment and a four-week run-in period with standard calcium and vitamin D supplementation, a single dose of denosumab (60 mg) was administered to all patients and follow-up visits were set at baseline, one day, one week and six months after the injection. Changes in PTH levels were the primary outcome of interest.

At baseline, both groups were comparable in age, calcium, albumin and PTH levels (Table 1). Mean serum calcium decreased in both groups at day 1 after denosumab injection from $10.0 \pm 0.4$ to $9.4 \pm 0.3$ $(\mathrm{p}<0.001)$ and $9.8 \pm 0.5$ to $9.01 \pm 0.4(\mathrm{p}<0.001)$ in patients with breast cancer and in controls, respectively. Overall mean serum PTH was significantly increased from 46.5 (31.1) to 62.3 (43.9) $\mathrm{pg} / \mathrm{mL}$ between baseline and day 7 in the total population studied (Wilcoxon Signed Rank test, $\mathrm{p}=0.007$ ) (data not shown). It should be noted that the PTH increase was evident only in the control group [70.1 (118.2) from 46.2 (62.2) pg/mL, Wilcoxon Signed Rank test: $\mathrm{p}=0.006]$ 
Table 1. Baseline characteristics of study population

\begin{tabular}{lccc}
\hline Variable & $\begin{array}{c}\text { Group A (Breast cancer) } \\
\mathbf{n = 2 1}\end{array}$ & $\begin{array}{c}\text { Group B (Controls) } \\
\mathbf{n = 2 5}\end{array}$ & p-value \\
\hline Age $($ mean \pm SD) & $58.1 \pm 8.9$ & $61.8 \pm 8.5$ & 0.134 \\
Serum Ca (mean \pm SD) & $10.0 \pm 0.4$ & $9.8 \pm 0.5$ & 0.169 \\
Serum P (mean \pm SD) & $3.7 \pm 0.5$ & $3.7 \pm 0.5$ & 0.694 \\
Serum PTH [median (IQR)] & $53.7(24.7)$ & $46.2(62.2)$ & $0.503 *$ \\
$\mathrm{U}_{\mathrm{Ca}}$ & $141(99)$ & $185(120)$ & $0.151 *$ \\
\hline
\end{tabular}

Variables were described with mean and standard deviation (SD) or median and IQR (interquartile range) according to their assessment of normality. Ca: Calcium, P: Phosphate, PTH: Parathyroid Hormone, $\mathrm{U}_{\mathrm{Ca}}$ : calcium in the $24 \mathrm{~h}$ urine collection.

Group A: Women with non-metastatic breast cancer and osteoporosis; Group B: Controls P-values are derived from T-test, unless otherwise specified. *Mann-Whitney test.

while in the breast cancer patients at day 7 was not significantly different from the mean PTH at baseline levels [53.2 (49.7) to 53.7 (24.7) $\mathrm{pg} / \mathrm{mL}$ following the intervention, $\mathrm{p}=0.394]$. On the other hand, mean serum PTH was decreased in the total study population from 62.3 (43.9) to $47.0(28.5) \mathrm{pg} / \mathrm{mL}$ (day 7 and day 180 respectively) (Wilcoxon Signed Rank test, $\mathrm{p}=0.099$ ). Interestingly, although non-significant in statistical terms, mean serum PTH was higher in breast cancer patients compared to controls at day 180 (Mann-Whitney test $\mathrm{p}=0.092$, Table 2). Moreover, a non-significant increase in mean serum PTH levels

Table 2. Changes in calcium homeostasis markers after denosumab injection.

\begin{tabular}{lcccc}
\hline & Ca & PTH & P & UCa \\
\hline Group A & & & & \\
\hline Baseline & $10.0 \pm 0.4$ & $53.7(24.7)$ & $3.8 \pm 0.4$ & $123 \pm 47$ \\
7 & $9.5 \pm 0.5$ & $53.2(49.7)$ & $3.3 \pm 0.6$ & $168 \pm 73$ \\
180 & $9.8 \pm 0.4 \mathrm{~b}$ & $60.9(34.5)$ & $3.6 \pm 0.4$ & $188 \pm 80$ \\
\hline Group B & & & & \\
\hline Baseline & $9.8 \pm 0.5$ & $46.2(62.2)$ & $3.6 \pm 0.5$ & $190(185)$ \\
7 & $9.2 \pm 0.5^{\mathrm{a}}$ & $70.1(118.2)^{\mathrm{a} *}$ & $3.1 \pm 0.5$ & $183(227)$ \\
180 & $9.6 \pm 0.3 \mathrm{~b}$ & $42.9(29.0)$ & $3.4 \pm 0.3$ & $164(134)$ \\
\hline
\end{tabular}

Ca: Calcium; P: Phosphate; PTH: Parathyroid Hormone; $\mathrm{U}_{\mathrm{Ca}}$ : calcium in the $24 \mathrm{~h}$ urine.

Group A: Women with non-metastastic breast cancer and osteoporosis, Group B: Controls.

aSignificant difference between baseline and day 7 measurements.

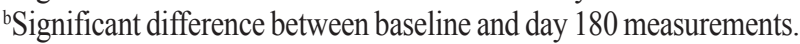
$\mathrm{P}$-values are derived from paierd T-test, unless otherwise specified. *Wilcoxon signed-rank test. was observed at day 180 in breast cancer patients in comparison to their baseline values (Figure 1).

In this pilot study, we confirm the short-term significant decrease in calcium levels in osteoporotic patients (with or without breast cancer) treated with denosumab. Moreover, an increase in PTH levels at 7 days was observed in the osteoporotic women with no breast cancer (controls) followed by a return to baseline levels at day 180 . However, in patients with breast cancer we noticed a delayed increase in PTH concentration at 180 days, probably as a response to the delayed significant decrease in calcium levels from baseline to 180 days.

The underlying pathophysiology to explain this different pattern of homeostatic calcium response between osteoporotic patients with breast cancer and osteoporotic controls remains to be elucidated. It could be speculated that this preliminary finding might be associated with a partial functional defect in CaSR, recently implicated in the pathogenesis of breast cancer. ${ }^{2}$ The mutations of BRCA1, which have been associated with hereditary breast cancer, were reported to function through CaSR by suppressing the expression of surviving and promoting sensitivity to paclitaxel. Moreover, CaSR has been involved in the dysregulation of intracellular calcium, which in turn has been associated with breast tumorigenesis. The above preliminary findings certainly warrant further investigation, though they should be considered in light of the study limitations, as the sample size was limited and the required six-month wash-out period may be short with regard to bisphosphonates and due 


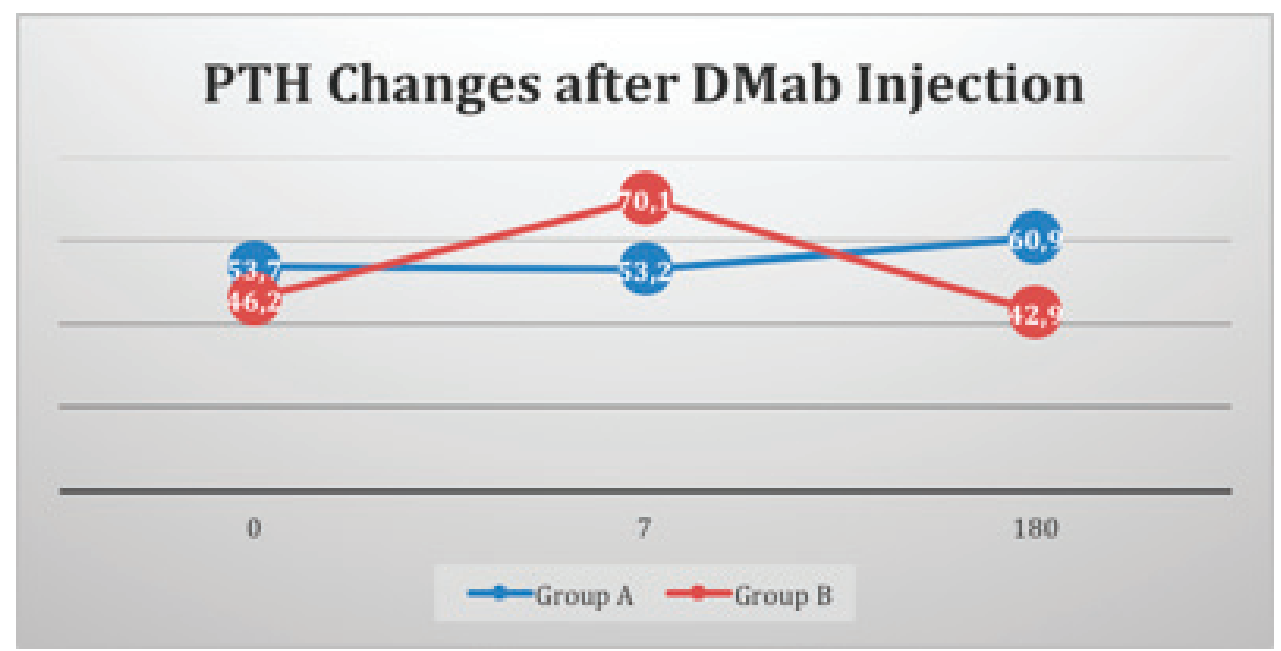

Figure 1. Parathyroid Hormone Changes (PTH) on day 7 and 180 after a single dose of Denosumab (DMab). Group A: patients with osteoporosis and non-metastatic breast cancer. Group B: Patients with osteoporosis.

to the lack of baseline Vitamin D, Mg and RANKL measurements in both groups.

Notwithstanding the above limitations, this study suggests that a blunted PTH response to acute RANKL inhibition induced by denosumab injection might be present in patients with breast cancer. If confirmed, this may have potentially important clinical and research implications. Specifically, if the dynamics of calcium and PTH response to acute RANKL inhibition are different, this could suggest that the efficacy of denosumab might also differ between patients with and without breast cancer. Furthermore, it would seem reasonable to consider a more aggressive and/ or prolonged approach in calcium and vitamin D supplementation in breast cancer patients receiving denosumab. Finally, the investigation of the relationship between changes in RANKL levels and the observed changes in Ca and PTH levels as well as a clarification of the role of CaSR in the latter would take us a step towards a deeper understanding of skeletal metabolism in patients with non-metastatic breast cancer.

To summarize, we have noted a pattern of delayed manifestation of secondary hyperparathyroidism present in osteoporotic women with non-metastatic breast cancer as compared to osteoporotic women without breast cancer. This observation warrants further investigation.

\section{REFERENCES}

1. Singh N, Promkan M, Liu G, et al, 2013 Role of calcium sensing receptor (CaSR) in tumorigenesis. Role of calcium sensing receptor (CaSR) in tumorigenesis. Best Pract Res Clin Endocrinol Metab 27: 455-463.

2. Sarkar P, Kumar S, 2012 Calcium sensing receptor modulation for cancer therapy. Asian Pac J Cancer Prev 13: 3561-3568. 